\title{
The complete convergence for weighted sums of dependent random fields
}

Mi Hwa Ko*

\section{"Correspondence:}

songhack@wonkwang.ac.kr

Division of Mathematics and Informational Statistics, Wonkwang

University, Jeonbuk, 570-749, Korea

\begin{abstract}
In this paper we extend the complete convergence for weighted sums of dependent random fields to the case that different indices have different powers in the normalization. Results are obtained for negatively associated random fields and $\rho^{*}$-mixing random fields.
\end{abstract}

MSC: 60F05; 60F15

Keywords: strong law of large numbers; complete convergence; negatively associated random field; $\rho^{*}$-mixing random field; weighted sums

\section{Introduction}

Let $Z_{+}^{d}(d \geq 2)$ denote the set of positive integer $d$-dimensional lattice points. For the elements of $Z_{+}^{d}$, we use bold symbols $\mathbf{m}, \mathbf{n}$ and define usual partial ordering for elements of $Z_{+}^{d}$, i.e., for $\mathbf{m}=\left(m_{1}, m_{2}, \ldots, m_{d}\right)$ and $\mathbf{n}=\left(n_{1}, n_{2}, \ldots, n_{d}\right), \mathbf{m} \leq \mathbf{n}$ if and only if $m_{i} \leq n_{i}$ for all $i=1,2, \ldots, d$. The strict inequality $\mathbf{m}<\mathbf{n}$ if and only if $\mathbf{m} \leq \mathbf{n}$ and $\mathbf{m} \neq \mathbf{n}$. We assume that $\mathbf{n} \rightarrow \infty$ means $\max _{1 \leq i \leq d} n_{i} \rightarrow \infty$ and use $|\mathbf{n}|$ for $\prod_{i=1}^{d} n_{i}$. In this work, $C$ indicates a generic positive number which may be different at different places, and $I(\cdot)$ indicates the indicator function.

Joag-Dev and Proschan [1] introduced the definition of negative association and showed that many of the well-known multivariate distributions possess the negatively associated property, for example, multivariate hypergeometric distribution, negatively correlated normal distribution, random sampling without replacement, etc. Also, they have showed that the class of negatively associated random variables is the only one among the different types of negative dependence which has the important property of being closed under formation of a nondecreasing function of disjoint sets of random variables.

Definition 1.1 (Joag-Dev, Proschan [1]) A random field $\left\{X_{\mathbf{n}}, \mathbf{n} \in Z_{+}^{d}\right\}$ is said to be negatively associated (NA) if for every pair of disjoint subsets $A$ and $B$ of $Z_{+}^{d}$ and any pair of coordinatewise nondecreasing functions $f$ and $g$,

$$
\operatorname{Cov}\left(f\left(X_{\mathbf{i}}, \mathbf{i} \in A\right), g\left(X_{\mathbf{j}}, \mathbf{j} \in B\right)\right) \leq 0
$$

whenever $f$ and $g$ are such that the covariance exists.

In many papers one can find some interesting results concerning the fields of negatively associated random variables. We refer only to some of them: Roussas [2] for the central

(c) $2013 \mathrm{Ko}$; licensee Springer. This is an Open Access article distributed under the terms of the Creative Commons Attribution License (http://creativecommons.org/licenses/by/2.0), which permits unrestricted use, distribution, and reproduction in any medium, provided the original work is properly cited. 
limit theorem for weakly stationary fields; Zhang and Wen [3] for the Rosenthal maximal inequality; Xia and Chu [4] for the convergence rates in the law of iterated logarithm and the Rosenthal maximal inequality for identically distributed random variables; Li [5] for the convergence rates in the law of iterated logarithm. One can find more interesting results in the recent monograph of Bulinski and Shashkin [6].

Peligrad and Gut [7] investigated a class of dependent random fields based on an interlaced condition which uses the maximal coefficient of correlation, and they defined the condition in the following way: Let $\left\{X_{\mathbf{n}}, \mathbf{n} \in Z_{+}^{d}\right\}$ be a random field, let $S \subset Z_{+}^{d}$ and define

$$
\begin{aligned}
\mathcal{F}_{S} & =\sigma\left(X_{\mathbf{i}}, \mathbf{i} \in S\right) \\
& =\text { the } \sigma \text {-field generated by the random variables }\left\{X_{\mathbf{i}}, \mathbf{i} \in S \subset Z_{+}^{d}\right\}
\end{aligned}
$$

and

$$
\begin{aligned}
\rho^{*}(k) & =\sup \operatorname{corr}(X, Y) \\
& =\sup _{S, T}\left(\sup _{X \in L^{2}\left(\mathcal{F}_{S}\right), Y \in L^{2}\left(\mathcal{F}_{T}\right)} \frac{|\operatorname{Cov}(X, Y)|}{(\operatorname{Var} X \operatorname{Var} Y)^{1 / 2}}\right),
\end{aligned}
$$

where the supremum is taken over all $S, T \subset Z_{+}^{d}$ with $\operatorname{dist}(S, T) \geq k$, and all $X \in L^{2}\left(\mathcal{F}_{S}\right)$, $Y \in L^{2}\left(\mathcal{F}_{T}\right)$ and

$$
\operatorname{dist}(S, T)^{2}=\inf _{x \in S, y \in T}\|x-y\|^{2}=\inf _{x \in S, y \in T} \sum_{i=1}^{n}\left(x_{i}-y_{i}\right)^{2},
$$

i.e., Euclidean distance. Various limit properties under condition $\rho^{*}(k) \rightarrow 0$ were studied by Bradley [8, 9] and Miller [10]. Bryc and Smolenski [11] and Peligrad [12] pointed out the importance of the condition

$$
\lim _{k \rightarrow \infty} \rho^{*}(k)<1
$$

in estimating the moments of partial sums or the maxima of partial sums. Let us also note that since $0 \leq \cdots \leq \rho^{*}(n) \leq \rho^{*}(n-1) \leq \cdots \leq \rho^{*}(1) \leq 1,(1.2)$ is equivalent to

$$
\rho^{*}(N)<1 \quad \text { for some } N \geq 1 .
$$

Definition 1.2 A random field $\left\{X_{\mathbf{n}}, \mathbf{n} \in Z_{+}^{d}\right\}$ is said to be $\rho^{*}$-mixing if (1.2) holds.

The $\rho^{*}$-mixing random variables were investigated by Bryc and Smolenski [11] (moment inequalities of partial sums), Bradley $[8,9]$ (equivalent mixing conditions and various limit properties), Peligrad [12] (a moment inequality of maximal partial sums for sequences) and Peligrad and Gut [7] (a moment inequality of maximal partial sums for fields and almost sure results of Marcinkiewicz-Zygmund type).

Recently, Kuczmaszewska and Lagodowski [13] proved the complete convergence for negatively associated random field and $\rho^{*}$-mixing random field.

Let $\left\{X_{\mathbf{n}}, \mathbf{n} \in Z_{+}^{d}\right\}$ be a field of real random variables, and let $\left\{a_{\mathbf{n}, \mathbf{k}}, \mathbf{n} \in Z_{+}^{d}, \mathbf{k} \in Z_{+}^{d}, \mathbf{k} \leq \mathbf{n}\right\}$ be an array of real numbers. The weighted sums in the form $\sum_{\mathbf{k} \leq \mathbf{n}} a_{\mathbf{n}, \mathbf{k}} X_{\mathbf{k}}$ can play an important role in various applied and theoretical problems, such as those of the least squares 
estimators (see Kafles and Bhaskara Rao [14]) and $M$-estimates (see Rao and Zhao [15]) in linear models, the nonparametric regression estimators (see Priestley and Chao [16]) etc. So, the study of the complete convergence for the weighted sums is very important and significant.

The aim of this paper is to extend a complete convergence of weighted sums $\sum_{\mathbf{i} \leq \mathbf{n}} a_{\mathbf{n}, \mathbf{i}} X_{\mathbf{i}}$ to the case that different indices have different powers in the normalization, where $\left\{a_{\mathbf{n}, \mathbf{i}}, \mathbf{n} \in Z_{+}^{d}, \mathbf{i} \leq \mathbf{n}\right\}$ is an array of real numbers and $\left\{X_{\mathbf{i}}, \mathbf{i} \in Z_{+}^{d}\right\}$ is a field of negatively associated random variables and $\rho^{*}$-mixing random variables.

We define $\boldsymbol{\alpha}=\left(\alpha_{1}, \alpha_{2}, \ldots, \alpha_{d}\right)$ and, without loss of generality, we assume that the coordinates are arranged in nondecreasing order so that $\alpha_{1}$ is the smallest one and $\alpha_{d}$ is the largest one. We further let $p$ denote the number of $\alpha$ 's which are equal to the smallest one, that is,

$$
p=\max \left\{k: \alpha_{k}=\alpha_{1}\right\}
$$

As is easily seen, the domain of interest concerning the $\alpha$ 's becomes

$$
\frac{1}{2} \leq \alpha_{1} \leq \alpha_{2} \leq \cdots \leq \alpha_{d} \leq 1
$$

where the boundary $\frac{1}{2}$ takes us into the realm of the central limit theorem and the boundary 1 corresponds to the Kolmogorov strong law.

\section{Remark}

(1) $\alpha_{1}=\alpha_{2}=\cdots=\alpha_{d}=\frac{1}{2}$ case: Let $\left\{X_{\mathbf{n}}, \mathbf{n} \in Z_{+}^{d}\right\}$ be a strictly stationary associated random field with $E X_{1}=0$ and $E X_{1}^{2}<\infty$. If $\sum_{\mathbf{n} \geq \mathbf{1}} \operatorname{Cov}\left(X_{\mathbf{1}}, X_{\mathbf{n}}\right)=1$, then $|\mathbf{n}|^{-\frac{1}{2}} S_{\mathbf{n}} \rightarrow^{\mathcal{D}} N(0,1)$, where $\rightarrow^{\mathcal{D}}$ means convergence in distribution and $S_{\mathbf{n}}=\sum_{\mathbf{i} \leq \mathbf{n}} X_{\mathbf{i}}$ (see Newman [17]).

(2) $\alpha_{1}=\alpha_{2}=\cdots=\alpha_{d}=1$ case: Let $\left\{X_{\mathbf{n}}, \mathbf{n} \in Z_{+}^{d}\right\}$ be a field of identically distributed and negatively associated random variables with $E X_{1}=0$. Then

$$
E\left|X_{\mathbf{1}}\right|\left(\log ^{+}\left|X_{\mathbf{1}}\right|\right)^{d-1}<\infty \quad \text { implies that } \frac{S_{\mathbf{n}}}{|\mathbf{n}|} \rightarrow 0 \text { a.s. }
$$

(see Ko [18]).

Finally, for ease of notation, we use the notation $\mathbf{n}^{\alpha}=\left(n_{1}^{\alpha_{1}}, n_{2}^{\alpha_{2}}, \ldots, n_{d}^{\alpha_{d}}\right)$ and $\left|\mathbf{n}^{\alpha}\right|=$ $\prod_{i=1}^{d} n_{i}^{\alpha_{i}}$

\section{Results}

We start this section with the following lemma useful in the proofs of the main results.

Lemma 2.1 (Kuczmaszewska and Lagodowski [13]) Let $\left\{X_{\mathbf{n}}, \mathbf{n} \in Z_{+}^{d}\right\}$ be a field of negatively associated random variables with $E X_{\mathbf{n}}=0$ for $\mathbf{n} \in Z_{+}^{d}$. Then, for $q \geq 2$, there exists a positive constant $C=C(q)$ such that, for all $\mathbf{n} \in Z_{+}^{d}$,

$$
E \max _{\mathbf{k} \leq \mathbf{n}}\left|S_{\mathbf{k}}\right|^{q} \leq C\left\{\left(\log _{2}|\mathbf{n}|\right)^{q d}\left(\sum_{\mathbf{k} \leq \mathbf{n}} E X_{\mathbf{k}}^{2}\right)^{q / 2}+\sum_{\mathbf{k} \leq \mathbf{n}} E\left|X_{\mathbf{k}}\right|^{q}\right\} .
$$


Now we consider the main result.

Theorem 2.2 Let $\left\{X_{\mathbf{n}}, \mathbf{n} \in Z_{+}^{d}\right\}$ be a negatively associated random field and let $\left\{a_{\mathbf{n}, \mathbf{i}}, \mathbf{n} \in\right.$ $\left.Z_{+}^{d}, \mathbf{1} \leq \mathbf{i} \leq \mathbf{n}\right\}$ be an array of weights. Let $\alpha=\left(\alpha_{1}, \alpha_{2}, \ldots, \alpha_{d}\right), \alpha_{1} r>1, \alpha_{1}>\frac{1}{2}$ and $\frac{1}{2} \leq \alpha_{1} \leq$ $\alpha_{2} \leq \cdots \leq 1$ and let for some $q \geq 2$,

(a) $\sum_{\mathbf{n}}|\mathbf{n}|^{\alpha_{1} r-2} \sum_{\mathbf{i} \leq \mathbf{n}} P\left(\left|a_{\mathbf{n}, \mathbf{i}} X_{\mathbf{i}}\right|>\left|\mathbf{n}^{\alpha}\right|\right)<\infty$,

(b) $\sum_{\mathbf{n}}|\mathbf{n}|^{\alpha_{1}(r-q)-2} \sum_{\mathbf{i} \leq \mathbf{n}}\left|a_{\mathbf{n}, \mathbf{i}}\right|^{q} E\left(\left|X_{\mathbf{i}}\right|^{q} I\left[\left|a_{\mathbf{n}, \mathbf{i}} X_{\mathbf{i}}\right| \leq\left|\mathbf{n}^{\alpha}\right|\right]\right)<\infty$,

(c) $\sum_{\mathbf{n}}|\mathbf{n}|^{\alpha_{1}(r-q)-2}\left(\log _{2}|\mathbf{n}|\right)^{q d}\left(\sum_{\mathbf{i}<\mathbf{n}} a_{\mathbf{n}, \mathbf{i}}^{2} E\left(X_{\mathbf{i}}^{2} I\left[\left|a_{\mathbf{n}, \mathbf{i}} X_{\mathbf{i}}\right| \leq\left|\mathbf{n}^{\alpha}\right|\right]\right)\right)^{\frac{q}{2}}<\infty$,

(d) $\max _{\mathbf{j} \leq \mathbf{n}}\left|\sum_{\mathbf{i} \leq \mathbf{j}} E\left(a_{\mathbf{n}, \mathbf{i}} X_{\mathbf{i}} I\left[\left|a_{\mathbf{n}, \mathbf{i}} X_{\mathbf{i}}\right| \leq\left|\mathbf{n}^{\alpha}\right|\right]\right)\right|=o\left(\left|\mathbf{n}^{\alpha}\right|\right)$,

where $\mathbf{n}^{\alpha}=\left(n_{1}^{\alpha_{1}}, n_{2}^{\alpha_{2}}, \ldots, n_{d}^{\alpha_{d}}\right)$ and $\left|\mathbf{n}^{\alpha}\right|=n_{1}^{\alpha_{1}} \times n_{2}^{\alpha_{2}} \times \cdots \times n_{d}^{\alpha_{d}}$.

Then, for all $\epsilon>0$,

$$
\sum_{\mathbf{n}}|\mathbf{n}|^{\alpha_{1} r-2} P\left\{\max _{\mathbf{j} \leq \mathbf{n}}\left|\sum_{\mathbf{i} \leq \mathbf{j}}\left(a_{\mathbf{n}, \mathbf{i}} X_{\mathbf{i}}\right)\right|>\epsilon\left|\mathbf{n}^{\alpha}\right|\right\}<\infty .
$$

Proof Let, for $\mathbf{1} \leq \mathbf{i} \leq \mathbf{n}$,

$$
\begin{aligned}
& X_{\mathbf{n}, \mathbf{i}}^{\prime}=X_{\mathbf{i}} I\left[\left|a_{\mathbf{n}, \mathbf{i}} X_{\mathbf{i}}\right| \leq\left|\mathbf{n}^{\alpha}\right|\right]+\frac{\left|\mathbf{n}^{\alpha}\right|}{a_{\mathbf{n}, \mathbf{i}}} I\left[a_{\mathbf{n}, \mathbf{i}} X_{\mathbf{i}}>\left|\mathbf{n}^{\alpha}\right|\right]-\frac{\left|\mathbf{n}^{\alpha}\right|}{a_{\mathbf{n}, \mathbf{i}}} I\left[a_{\mathbf{n}, \mathbf{i}} X_{\mathbf{i}}<-\left|\mathbf{n}^{\alpha}\right|\right], \\
& S_{\mathbf{n}, \mathbf{i}}^{\prime}=\sum_{\mathbf{1} \leq \mathbf{i} \leq \mathbf{n}}\left(a_{\mathbf{n}, \mathbf{i}} X_{\mathbf{n}, \mathbf{i}}^{\prime}-E a_{\mathbf{n}, \mathbf{i}} X_{\mathbf{n}, \mathbf{i}}^{\prime}\right) .
\end{aligned}
$$

Let us notice that if the series $\sum_{\mathbf{n}}|\mathbf{n}|^{\alpha_{1} r-2}$ is convergent, then (2.2) always holds. Therefore we consider only the case such that $\sum_{\mathbf{n}}|\mathbf{n}|^{\alpha_{1} r-2}$ is divergent.

Let us observe that using the Chebyshev inequality we get

$$
\begin{aligned}
& P\left[\max _{\mathbf{j} \leq \mathbf{n}}\left|\sum_{\mathbf{i} \leq \mathbf{j}}\left(\left|\mathbf{n}^{\alpha}\right| I\left[\left|a_{\mathbf{n}, \mathbf{i}} X_{\mathbf{i}}\right|>\left|\mathbf{n}^{\alpha}\right|\right]+\left|\mathbf{n}^{\alpha}\right| P\left[\left|a_{\mathbf{n}, \mathbf{i}} X_{\mathbf{i}}\right|>\left|\mathbf{n}^{\alpha}\right|\right]\right)\right|>\left|\mathbf{n}^{\alpha}\right| \frac{\epsilon}{2}\right] \\
& \quad \leq P\left[\sum_{\mathbf{i} \leq \mathbf{n}}\left(I\left[\left|a_{\mathbf{n}, \mathbf{i}} X_{\mathbf{i}}\right|>\left|\mathbf{n}^{\alpha}\right|\right]+P\left[\left|a_{\mathbf{n}, \mathbf{i}} X_{\mathbf{i}}\right|>\left|\mathbf{n}^{\alpha}\right|\right]\right)>\frac{\epsilon}{2}\right] \\
& \quad \leq \frac{4}{\epsilon} \sum_{\mathbf{i} \leq \mathbf{n}} P\left[\left|a_{\mathbf{n}, \mathbf{i}} X_{\mathbf{i}}\right|>\left|\mathbf{n}^{\alpha}\right|\right] .
\end{aligned}
$$

By simple computations, using (2.3) and the assumptions (a) and (d), we obtain the following estimation:

$$
\begin{aligned}
P\left[\max _{\mathbf{j} \leq \mathbf{n}}\left|\sum_{\mathbf{i} \leq \mathbf{j}}\left(a_{\mathbf{n}, \mathbf{i}} X_{\mathbf{i}}\right)\right|>\epsilon\left|\mathbf{n}^{\alpha}\right|\right] \\
\leq P\left[\max _{\mathbf{j} \leq \mathbf{n}}\left|\sum_{\mathbf{i} \leq \mathbf{j}} a_{\mathbf{n}, \mathbf{i}} X_{\mathbf{i}}\right|>\epsilon\left|\mathbf{n}^{\alpha}\right|,\left|a_{\mathbf{n}, \mathbf{1}} X_{\mathbf{1}}\right| \leq\left|\mathbf{n}^{\alpha}\right|, \ldots,\left|a_{\mathbf{n}, \mathbf{n}} X_{\mathbf{n}}\right| \leq\left|\mathbf{n}^{\alpha}\right|\right] \\
\quad+P\left[\max _{\mathbf{j} \leq \mathbf{n}}\left|\sum_{\mathbf{i} \leq \mathbf{j}} a_{\mathbf{n}, \mathbf{i}} X_{\mathbf{i}}\right|>\epsilon\left|\mathbf{n}^{\alpha}\right|,\left|a_{\mathbf{n}, \mathbf{1}} X_{\mathbf{1}}\right|>\left|\mathbf{n}^{\alpha}\right| \cup \cdots \cup\left|a_{\mathbf{n}, \mathbf{n}} X_{\mathbf{n}}\right|>\left|\mathbf{n}^{\alpha}\right|\right] \\
\quad \leq P\left[\max _{\mathbf{j} \leq \mathbf{n}}\left|\sum_{\mathbf{i} \leq \mathbf{j}} a_{\mathbf{n}, \mathbf{i}} X_{\mathbf{i}} I\left[\left|a_{\mathbf{n}, \mathbf{i}} X_{\mathbf{i}}\right| \leq\left|\mathbf{n}^{\alpha}\right|\right]\right|>\epsilon\left|\mathbf{n}^{\alpha}\right|\right]+\sum_{\mathbf{i} \leq \mathbf{n}} P\left[\left|a_{\mathbf{n}, \mathbf{i}} X_{\mathbf{i}}\right|>\left|\mathbf{n}^{\alpha}\right|\right]
\end{aligned}
$$




$$
\begin{aligned}
\leq & P\left\{\max _{\mathbf{j} \leq \mathbf{n}}\left|S_{\mathbf{n}, \mathbf{j}}^{\prime}\right|>\epsilon\left|\mathbf{n}^{\alpha}\right|-\sum_{\mathbf{i} \leq \mathbf{n}}\left|\mathbf{n}^{\alpha}\right|\left(I\left[\left|a_{\mathbf{n}, \mathbf{i}} X_{\mathbf{i}}\right|>\left|\mathbf{n}^{\alpha}\right|\right]+P\left[\left|a_{\mathbf{n}, \mathbf{i}} X_{\mathbf{i}}\right|>\left|\mathbf{n}^{\alpha}\right|\right]\right)\right. \\
& \left.-\max _{\mathbf{j} \leq \mathbf{n}}\left|\sum_{\mathbf{i} \leq \mathbf{j}} E\left(a_{\mathbf{n}, \mathbf{i}} X_{\mathbf{i}} I\left[\left|a_{\mathbf{n}, \mathbf{i}} X_{\mathbf{i}}\right| \leq\left|\mathbf{n}^{\alpha}\right|\right]\right)\right|\right\}+\sum_{\mathbf{i} \leq \mathbf{n}} P\left[\left|a_{\mathbf{n}, \mathbf{i}} X_{\mathbf{i}}\right|>\left|\mathbf{n}^{\alpha}\right|\right] \\
\leq & P\left\{\max _{\mathbf{j} \leq \mathbf{n}}\left|S_{\mathbf{n}, \mathbf{j}}^{\prime}\right|+\max _{\mathbf{j} \leq \mathbf{n}} \sum_{\mathbf{i} \leq \mathbf{j}}\left|\mathbf{n}^{\alpha}\right|\left(I\left[\left|a_{\mathbf{n}, \mathbf{i}} X_{\mathbf{i}}\right|>\left|\mathbf{n}^{\alpha}\right|\right]+P\left[\left|a_{\mathbf{n}, \mathbf{i}} X_{\mathbf{i}}\right|>\left|\mathbf{n}^{\alpha}\right|\right]\right) \geq \epsilon\left|\mathbf{n}^{\alpha}\right|\right. \\
& \left.-\max _{\mathbf{j} \leq \mathbf{n}}\left|\sum_{\mathbf{i} \leq \mathbf{j}} E\left(a_{\mathbf{n}, \mathbf{i}} X_{\mathbf{i}} I\left[\left|a_{\mathbf{n}, \mathbf{i}} X_{\mathbf{i}}\right| \leq\left|\mathbf{n}^{\alpha}\right|\right]\right)\right|\right\}+\sum_{\mathbf{i} \leq \mathbf{n}} P\left[\left|a_{\mathbf{n}, \mathbf{i}} X_{\mathbf{i}}\right|>\left|\mathbf{n}^{\alpha}\right|\right] \\
\leq & P\left\{\max _{\mathbf{j} \leq \mathbf{n}}\left|S_{\mathbf{n}, \mathbf{j}}^{\prime}\right|>\left|\mathbf{n}^{\alpha}\right| \frac{\epsilon}{2}-\max _{\mathbf{j} \leq \mathbf{n}}\left|\sum_{\mathbf{i} \leq \mathbf{j}} E\left(a_{\mathbf{n}, \mathbf{i}} X_{\mathbf{i}} I\left[\left|a_{\mathbf{n}, \mathbf{i}} X_{\mathbf{i}}\right| \leq\left|\mathbf{n}^{\alpha}\right|\right]\right)\right|\right\} \\
& +P\left\{\max _{\mathbf{j} \leq \mathbf{n}}\left|\sum_{\mathbf{i} \leq \mathbf{j}}\right| \mathbf{n}^{\alpha}\left|\left(I\left[\left|a_{\mathbf{n}, \mathbf{i}} X_{\mathbf{i}}\right|>\left|\mathbf{n}^{\alpha}\right|\right]+P\left[\left|a_{\mathbf{n}, \mathbf{i}} X_{\mathbf{i}}\right|>\left|\mathbf{n}^{\alpha}\right|\right]\right)\right|>\left|\mathbf{n}^{\alpha}\right| \frac{\epsilon}{2}\right\} \\
& +\sum_{\mathbf{i} \leq \mathbf{n}} P\left[\left|a_{\mathbf{n}, \mathbf{i}} X_{\mathbf{i}}\right|>\left|\mathbf{n}^{\alpha}\right|\right] \\
\leq & P\left[\max _{\mathbf{j} \leq \mathbf{n}}\left|S_{\mathbf{n}, \mathbf{j}}^{\prime}\right|>\left|\mathbf{n}^{\alpha}\right| \frac{\epsilon}{4}\right]+\left(\frac{4}{\epsilon}+1\right) \sum_{\mathbf{i} \leq \mathbf{n}} P\left[\left|a_{\mathbf{n}, \mathbf{i}} X_{\mathbf{i}}\right|>\left|\mathbf{n}^{\alpha}\right|\right] \quad \text { for all } \epsilon>0 .
\end{aligned}
$$

Note that the last inequality of (2.4) is obtained by (2.3) and (d).

Let $C$ in our further consideration denote a positive constant which changes from one appearance to the next.

Using the $C_{r}$ inequality, we can estimate that

$$
\begin{aligned}
E \mid & a_{\mathbf{n}, \mathbf{i}} X_{\mathbf{n}, \mathbf{i}}^{\prime}-\left.E a_{\mathbf{n}, \mathbf{i}} X_{\mathbf{n}, \mathbf{i}}^{\prime}\right|^{q} \\
& \leq C E\left|a_{\mathbf{n}, \mathbf{i}} X_{\mathbf{n}, \mathbf{i}}^{\prime}\right|^{q} \\
& =C\left\{E\left(\left|a_{\mathbf{n}, \mathbf{i}} X_{\mathbf{i}}\right|^{q} I\left[\left|a_{\mathbf{n}, \mathbf{i}} X_{\mathbf{i}}\right| \leq\left|\mathbf{n}^{\alpha}\right|\right]\right)+\left|\mathbf{n}^{\alpha}\right|^{q} P\left(\left|a_{\mathbf{n}, \mathbf{i}} X_{\mathbf{i}}\right|>\left|\mathbf{n}^{\alpha}\right|\right)\right\} .
\end{aligned}
$$

Thus, by (2.4), the Chebyshev inequality, Lemma 2.1 and (2.5), we get

$$
\begin{aligned}
P\left[\max _{\mathbf{j} \leq \mathbf{n}}\left|\sum_{\mathbf{i} \leq \mathbf{j}}\left(a_{\mathbf{n}, \mathbf{i}} X_{\mathbf{i}}\right)\right|>\epsilon\left|\mathbf{n}^{\alpha}\right|\right] \\
\leq P\left[\max _{\mathbf{j} \leq \mathbf{n}}\left|S_{\mathbf{n}, \mathbf{j}}^{\prime}\right|>\left|\mathbf{n}^{\alpha}\right| \frac{\epsilon}{4}\right]+\left(1+\frac{4}{\epsilon}\right) \sum_{\mathbf{i} \leq \mathbf{n}} P\left[\left|a_{\mathbf{n}, \mathbf{i}} X_{\mathbf{i}}\right|>\left|\mathbf{n}^{\alpha}\right|\right] \\
\leq \frac{C}{\left|\mathbf{n}^{\alpha}\right|^{q}} E \max _{j \leq n}\left|S_{\mathbf{n}, \mathbf{j}}^{\prime}\right|^{q}+\left(1+\frac{4}{\epsilon}\right) \sum_{\mathbf{i} \leq \mathbf{n}} P\left[\left|a_{\mathbf{n}, \mathbf{i}} X_{\mathbf{i}}\right|>\left|\mathbf{n}^{\alpha}\right|\right] \\
\leq \frac{C}{\left|\mathbf{n}^{\alpha}\right|^{q}}\left\{\left(\log _{2}|\mathbf{n}|\right)^{q d}\left(\sum_{\mathbf{i} \leq \mathbf{n}} E\left(a_{\mathbf{n}, \mathbf{i}} X_{\mathbf{n}, \mathbf{i}}^{\prime}-E a_{\mathbf{n}, \mathbf{i}} X_{\mathbf{n}, \mathbf{i}}^{\prime}\right)^{2}\right)^{q / 2}\right. \\
\left.\quad+\sum_{\mathbf{i} \leq \mathbf{n}} E\left|a_{\mathbf{n}, \mathbf{i}} X_{n, i}^{\prime}-E a_{\mathbf{n}, \mathbf{i}} X_{\mathbf{n}, \mathbf{i}}^{\prime}\right|^{q}\right\}+\left(1+\frac{4}{\epsilon}\right) \sum_{\mathbf{i} \leq \mathbf{n}} P\left[\left|a_{\mathbf{n}, \mathbf{i}} X_{\mathbf{i}}\right|>\left|\mathbf{n}^{\alpha}\right|\right] \\
\leq \frac{C}{\left|\mathbf{n}^{\alpha}\right|^{q}}\left\{\left(\log _{2}|\mathbf{n}|\right)^{q d}\left(\sum_{\mathbf{i} \leq \mathbf{n}} E a_{\mathbf{n}, \mathbf{i}}^{2} X_{\mathbf{n}, \mathbf{i}}^{\prime}\right)^{q / 2}+\sum_{\mathbf{i} \leq \mathbf{n}} E\left|a_{\mathbf{n}, \mathbf{i}} X_{n, i}^{\prime}\right|^{q}\right\}
\end{aligned}
$$




$$
\begin{aligned}
& +\left(1+\frac{4}{\epsilon}\right) \sum_{\mathbf{i} \leq \mathbf{n}} P\left[\left|a_{\mathbf{n}, \mathbf{i}} X_{\mathbf{i}}\right|>\left|\mathbf{n}^{\alpha}\right|\right] \\
\leq & C\left\{| \mathbf { n } ^ { \alpha } | ^ { - q } \left[\left(\log _{2}|\mathbf{n}|\right)^{q d}\left(\sum_{\mathbf{i} \leq \mathbf{n}} E\left(a_{\mathbf{n}, \mathbf{i}}^{2} X_{\mathbf{i}}^{2} I\left[\left|a_{\mathbf{n}, \mathbf{i}} X_{\mathbf{i}}\right| \leq\left|\mathbf{n}^{\alpha}\right|\right]\right)\right)^{q / 2}\right.\right. \\
& \left.\left.+\sum_{\mathbf{i} \leq \mathbf{n}} E\left(\left|a_{\mathbf{n}, \mathbf{i}} X_{\mathbf{i}}\right|^{q} I\left[\left|a_{\mathbf{n}, \mathbf{i}} X_{\mathbf{i}}\right| \leq\left|\mathbf{n}^{\alpha}\right|\right]\right)\right]+\sum_{\mathbf{i} \leq \mathbf{n}} P\left[\left|a_{\mathbf{n}, \mathbf{i}} X_{\mathbf{i}}\right|>\left|\mathbf{n}^{\alpha}\right|\right]\right\} \\
\leq & C\left\{| \mathbf { n } | ^ { - \alpha _ { 1 } q } \left[\left(\log _{2}|\mathbf{n}|\right)^{q d}\left(\sum_{\mathbf{i} \leq \mathbf{n}} E\left(a_{\mathbf{n}, \mathbf{i}}^{2} X_{\mathbf{i}}^{2} I\left[\left|a_{\mathbf{n}, \mathbf{i}} X_{\mathbf{i}}\right| \leq\left|\mathbf{n}^{\alpha}\right|\right]\right)\right)^{q / 2}\right.\right. \\
& \left.\left.+\sum_{\mathbf{i} \leq \mathbf{n}} E\left(\left|a_{\mathbf{n}, \mathbf{i}} X_{\mathbf{i}}\right|^{q} I\left[\left|a_{\mathbf{n}, \mathbf{i}} X_{\mathbf{i}}\right| \leq\left|\mathbf{n}^{\alpha}\right|\right]\right)\right]+\sum_{\mathbf{i} \leq \mathbf{n}} P\left[\left|a_{\mathbf{n}, \mathbf{i}} X_{\mathbf{i}}\right|>\left|\mathbf{n}^{\alpha}\right|\right]\right\} \\
& \text { since } \frac{1}{2} \leq \alpha_{1} \leq \alpha_{2} \leq \cdots \leq \alpha_{d} \leq 1 .
\end{aligned}
$$

Therefore, it follows from (a), (b), (c) and (2.6), that (2.2) holds. This completes the proof of Theorem 2.2.

Remark In the case $q=2$, the assumptions (b) and (c) reduce to

(b') $\sum_{\mathbf{n}}|\mathbf{n}|^{\alpha_{1}(r-2)-2}\left(\log _{2}|\mathbf{n}|\right)^{2 d} \sum_{\mathbf{i} \leq \mathbf{n}} E\left(a_{\mathbf{n}, \mathbf{i}}^{2} X_{\mathbf{i}}^{2} I\left[\left|a_{\mathbf{n}, \mathbf{i}} X_{\mathbf{i}}\right| \leq\left|\mathbf{n}^{\alpha}\right|\right]\right)<\infty$.

Letting $\alpha_{1}=\alpha_{2}=\cdots=\alpha_{d}=\alpha$ and $a_{\mathbf{n}, \mathbf{i}}=1$ for all $\mathbf{n} \in Z_{+}^{d}, \mathbf{1} \leq \mathbf{i} \leq \mathbf{n}$ from Theorem 2.2, the following corollary (Theorem 2.1 of [13]) is obtained.

Corollary 2.3 (Kuczmaszewska and Lagodowski [13]) Let $\left\{X_{\mathbf{n}}, \mathbf{n} \in Z_{+}^{d}\right\}$ be a field of negatively associated random variables. Let $\alpha r>1, \alpha>\frac{1}{2}$ and for some $q \geq 2$,

(i) $\sum_{\mathbf{n}}|\mathbf{n}|^{\alpha r-2} \sum_{\mathbf{i} \leq \mathbf{n}} P\left(\left|X_{\mathbf{i}}\right|>|\mathbf{n}|^{\alpha}\right)<\infty$,

(ii) $\sum_{\mathbf{n}}|\mathbf{n}|^{\alpha(r-q)-2} \sum_{\mathbf{i} \leq \mathbf{n}} E\left(\left|X_{\mathbf{i}}\right|^{q} I\left[\left|X_{\mathbf{i}}\right| \leq|\mathbf{n}|^{\alpha}\right]\right)<\infty$,

(iii) $\sum_{\mathbf{n}}|\mathbf{n}|^{\alpha(r-q)-2}\left(\log _{2}|\mathbf{n}|\right)^{q d}\left(\sum_{\mathbf{i} \leq \mathbf{n}} E\left(X_{\mathbf{i}}^{2} I\left[\left|X_{\mathbf{i}}\right| \leq|\mathbf{n}|^{\alpha}\right]\right)\right)^{\frac{q}{2}}<\infty$,

(iv) $\max _{\mathbf{j} \leq \mathbf{n}}\left|\sum_{\mathbf{i} \leq \mathbf{j}} E\left(X_{\mathbf{i}} \mathbf{I}\left[\left|X_{\mathbf{i}}\right| \leq|\mathbf{n}|^{\alpha}\right]\right)\right|=o\left(|\mathbf{n}|^{\alpha}\right)$.

Then

$$
\sum_{\mathbf{n}}|\mathbf{n}|^{\alpha r-2} P\left\{\max _{\mathbf{j} \leq \mathbf{n}}\left|S_{\mathbf{j}}\right|>\epsilon|\mathbf{n}|^{\alpha}\right\}<\infty
$$

for all $\epsilon>0$, where $S_{\mathbf{j}}=\sum_{\mathbf{i} \leq \mathbf{j}} X_{\mathbf{i}}$

Next we extend the complete convergence for weighted sums of $\rho^{*}$-mixing random field to the case that indices have different powers in the normalization.

Lemma 2.4 (Peligrad and Gut [7]) Let $\left\{X_{\mathbf{n}}, \mathbf{n} \in Z_{+}^{d}\right\}$ be a field of $\rho^{*}$-mixing random variables satisfying (1.2), $E X_{\mathbf{n}}=0$ and $E\left|X_{\mathbf{n}}\right|^{q}<\infty$ for $q \geq 2$ and $\mathbf{n} \in Z_{+}^{d}$. Then there exist positive constants $K_{1}=K_{1}\left(q, \rho^{*}(N), d\right)$ and $K_{2}=K_{2}\left(q, \rho^{*}(N), d\right)$ such that

$$
E \max _{\mathbf{k} \leq \mathbf{n}}\left|S_{\mathbf{k}}\right|^{q} \leq K_{1}\left\{\sum_{\mathbf{k} \leq \mathbf{n}} E\left|X_{\mathbf{k}}\right|^{q}+\left(\log _{2}|\mathbf{n}|\right)^{q d}\left(\sum_{\mathbf{k} \leq \mathbf{n}} E X_{\mathbf{k}}^{2}\right)^{q / 2}\right\} \quad \text { for all } \mathbf{n} \in Z_{+}^{d}
$$


and

$$
E\left|S_{\mathbf{k}}\right|^{q} \leq K_{2} E\left(\sum_{\mathbf{k} \leq \mathbf{n}} X_{\mathbf{k}}^{2}\right)^{q / 2} \quad \text { for all } \mathbf{n} \in Z_{+}^{d} .
$$

We are going to generalize the result given by Kuczmaszewska and Lagodowski [13, Theorem 3.1] to the case that different indices have different powers in the normalization.

Theorem 2.5 Let $\left\{X_{\mathbf{n}}, \mathbf{n} \in Z_{+}^{d}\right\}$ be a field of $\rho^{*}$-mixing random variables with $E X_{\mathbf{n}}=0$ and let $\left\{a_{\mathbf{n}, \mathbf{i}}, \mathbf{n} \in Z_{+}^{d}, \mathbf{1} \leq \mathbf{i} \leq \mathbf{n}\right\}$ be an array of real numbers. Let $\alpha_{1} r>1, \alpha_{1}>\frac{1}{2}$ and let for some $q \geq 2$, (a)-(d) in Theorem 2.2 be satisfied. Then (2.2) holds.

Proof Let, for $\mathbf{1} \leq \mathbf{i} \leq \mathbf{n}$,

$$
\begin{aligned}
& X_{\mathbf{n}, \mathbf{i}}^{\prime}=X_{\mathbf{i}} I\left[\left|a_{\mathbf{n}, \mathbf{i}} X_{\mathbf{i}}\right| \leq\left|\mathbf{n}^{\alpha}\right|\right], \\
& Y_{\mathbf{n}, \mathbf{i}}=a_{\mathbf{n}, \mathbf{i}} X_{\mathbf{n}, \mathbf{i}}^{\prime}-a_{\mathbf{n}, \mathbf{i}} E X_{\mathbf{n}, \mathbf{i}}^{\prime} \text { and } S_{\mathbf{n}, \mathbf{k}}^{\prime}=\sum_{\mathbf{i} \leq \mathbf{k}} Y_{\mathbf{n}, \mathbf{i}} .
\end{aligned}
$$

By (2.8) we obtain (2.2) arguing as before in the proof of Theorem 2.2.

Letting $\alpha_{1}=\alpha_{2}=\cdots=\alpha_{d}=1$ from Theorem 2.5, the following corollary (Theorem 3.1 of [13]) is obtained.

Corollary 2.6 Let $\left\{X_{\mathbf{n}}, \mathbf{n} \in Z_{+}^{d}\right\}$ be a field of random variables satisfying (1.2). Let $\alpha r>1$, $\alpha>\frac{1}{2}$ and, for some $q \geq 2$, (i)-(iv) of Corollary 2.3 be satisfied. Then (2.7) holds.

\section{Competing interests}

The author declares that she has no competing interests.

\section{Acknowledgements}

The author is very grateful to the referees for careful reading of the manuscript and for the valuable suggestions which allowed her to improve the paper. This research was supported by Basic Science Research Program through the National Research Foundation of Korea (NRF) funded by the Ministry of Education, Science and Technology (2012R1A1A3008230).

Received: 11 February 2013 Accepted: 10 July 2013 Published: 26 July 2013

\section{References}

1. Joag-Dev, K, Proschan, F: Negative association of random variables with applications. Ann. Stat. 11, $286-295$ (1983)

2. Roussas, G: Asymptotic normality of random fields of positively or negatively associated processes. J. Multivar. Anal. 50, 152-173 (1994)

3. Zhang, LX, Wen, J: A weak convergence for negatively associated fields. Stat. Probab. Lett. 53, 259-267 (2001)

4. Xia, W, Chu, Y: The convergence of the logarithm involving negatively associated random fields. Appl. Math. Sci. 3, 667-675 (2009)

5. Li, YX: Convergence rates in the law of iterated logarithm for negatively associated random variables with multidimensional indices. Stat. Probab. Lett. 79, 1038-1043 (2009)

6. Bulinski, A, Shashkin, A: Limit Theorems for Associated Random Fields and Related Systems. World Scientific, Hackensack (2007)

7. Peligrad, M, Gut, A: Almost sure results for class of dependent random variables. J. Theor. Probab. 12, 87-104 (1999)

8. Bradley, RC: On the spectral density and asymptotic normality of weakly dependent random fields. J. Theor. Probab. 5, 355-373 (1992)

9. Bradley, RC: Equivalent mixing conditions for random fields. Ann. Probab. 21, 1921-1926 (1993)

10. Miller, C: Three theorems of $\rho^{*}$-mixing random fields. J. Theor. Probab. 7, 867-882 (1994)

11. Bryc, W, Smolenski, W: Moment conditions for almost sure convergence of weakly correlated random variables. Proc. Am. Math. Soc. 119, 629-635 (1993)

12. Peligrad, M: Maximum of partial sums and an invariance principle for a class of weakly dependent random variables. Proc. Am. Math. Soc. 126, 1181-1189 (1998) 
13. Kuczmaszewska, A, Lagodowski, ZA: Convergence rates in the SLLN for some classes of dependent random field. J. Math. Anal. Appl. 380, 571-584 (2011)

14. Kafles, D, Bhaskara Rao, M: Weak consistency of least squares estimators in linear models. J. Multivar. Anal. 12, 186-198 (1982)

15. Rao, CR, Zhao, LC: Linear representation of M-estimates in linear models. Can. J. Stat. 20, 359-368 (1992)

16. Priestley, MB, Chao, MT: Nonparametric function fitting. J. R. Stat. Soc. B 34, 385-392 (1972)

17. Newman, CM: Normal fluctuations and the FKG inequalities. Commun. Math. Phys. 74, 119-128 (1980)

18. Ko, MH: On the complete convergence for negatively associated random fields. Taiwan. J. Math. 15, 165-169 (2011)

doi:10.1186/1029-242X-2013-348

Cite this article as: Ko: The complete convergence for weighted sums of dependent random fields. Journal of Inequalities and Applications 2013 2013:348.

Submit your manuscript to a SpringerOpen ${ }^{\circ}$ journal and benefit from:

- Convenient online submission

- Rigorous peer review

- Immediate publication on acceptance

- Open access: articles freely available online

- High visibility within the field

- Retaining the copyright to your article

Submit your next manuscript at $>$ springeropen.com 